\title{
An assessment of medical students' awareness of radiation exposures associated with diagnostic imaging investigations
}

\author{
Jennifer O'Sullivan • Owen J. O'Connor • \\ Kevin O'Regan • Bronagh Clarke • \\ Louise N. Burgoyne • Max F. Ryan • Michael M. Maher
}

Received: 18 November 2009 / Accepted: 30 November 2009/Published online: 3 March 2010

(C) European Society of Radiology 2010

\begin{abstract}
Objectives This study assessed students' awareness of radiation exposures and determined the impact a curriculum in clinical radiology (CICR) had on awareness.

Methods Six hundred seventy medical students at one medical school were studied. CICR was delivered in yearly modules over the 5-year programme. Five hundred twentythree students (years 1-5), exposed to increasing numbers of CICR modules and 147 students beginning medical school (year 0), represented the study and control groups, respectively. Students completed a multiple choice questionnaire assessing radiation knowledge and radiology teaching.

Results Most students in the study population received CICR but $87 \%$ considered they had not received radiation protection instruction. The percentage of correctly answered questions was significantly higher in the study population than the control group $(59.7 \%$ versus $38 \%, \mathrm{p}<0.001)$. Students who received CICR achieved higher scores than those who did not $(61.3 \%$ compared with $42.8 \%, \mathrm{p}<0.001)$. Increasing exposure to CICR with each year of medical education was associated with improved performance.

Conclusions Assessment of students' awareness of radiation exposures in diagnostic imaging demonstrates improved performance with increasing years in medical school and/or increasing exposure to CICR. Findings support the Euroatom 97 directive position, advocating implementation of radiation protection instruction into the undergraduate medical curriculum.
\end{abstract}

J. O’Sullivan · O. J. O’Connor $\cdot$ K. O'Regan · B. Clarke ·

M. F. Ryan • M. M. Maher $(\bowtie)$

Department of Radiology, University College Cork,

Cork, Ireland

e-mail: m.maher@ucc.ie

L. N. Burgoyne

Department of Epidemiology, University College Cork,

Cork, Ireland
Keywords Medical students · Radiation protection . Medical education

\section{Introduction}

In recent years, studies have documented increasing patient radiation exposures, which can be attributed to increased utilisation of diagnostic imaging studies, particularly computed tomography (CT). For example, a retrospective study of radiation doses in a cohort of patients with Crohn's disease conducted over a 15 -year period demonstrated that increasing numbers of CT exams were performed with average cumulative effective doses rising from 7.9 to $25 \mathrm{mSv}$ when the first 5 years of the study period were compared with the final 5 years [1]. Eighty-five percent of the radiation dose during the final 5 -year period was attributable to CT. Almost $16 \%$ of patients received cumulative effective doses greater than $75 \mathrm{mSv}$, and such levels of radiation exposure have been reported to be associated with a $7.3 \%$ increase in mortality from cancer [2]. In a separate study, CT has been shown to account for merely $6 \%$ of diagnostic procedures, yet represented $47 \%$ of the entire radiation dose received by patients [3]. Furthermore, the number of referrals for paediatric CT studies has increased exponentially, raising substantial concerns regarding cancer risk in this highly radiosensitive population [4]. Not surprisingly, concerns are growing over the risks associated with these high levels of exposure, particularly the potential increased lifetime risk of cancer [5].

This issue has been addressed by the European Council Eurotom directive of 1997, which made a number of recommendations. Among the recommendations were that radiation protection should be integrated into the curriculum of medical schools [6]. Concerns about radiation exposure have also precipitated the formation of Image Gently, a campaign in the US aimed at increasing public 
awareness of radiation exposure in children [7, 8]. Accordingly, it is imperative that medical professionals are aware of the radiation exposures associated with diagnostic imaging investigations including $\mathrm{CT}$.

Over the past decade many studies have investigated physicians from varying specialties and backgrounds on their knowledge of radiation dose and associated risks, and most studies have demonstrating disappointing results [9-11]. Most physicians significantly underestimated doses associated with various imaging modalities. Although studies of medical students' knowledge of radiology have been conducted and reported, the specific area of awareness among medical students of relative radiation doses associated with different diagnostic imaging investigations and assumed cancer risk has yet to be thoroughly investigated $[12,13]$.

This study aimed to assess students' awareness of relative radiation exposures associated with common diagnostic imaging procedures and to determine the impact of a curriculum in clinical radiology (CICR) in the undergraduate medical programme on such awareness.

\section{Materials and methods}

Following institutional review board approval, a multiple choice questionnaire was distributed to the study population comprising 1 st to 5 th year medical students $(n=523)$ at the completion of the academic year (April 2008), and to a control group, Year 0 [1st year medical students $(n=147)$ on their first week at medical school (September 2008)] during orientation and prior to receiving CICR (Appendix). Year 0, comprised two different groups: students admitted directly from high school who had yet to receive any teaching in radiology or radiation protection in a post-graduate setting and who would go on to complete a 5-year undergraduate medical programme, and Graduate Entry Medical (GEM) students (students who had completed a university degree and had achieved at least 2 nd honours grade). This was the first year of the GEM programme at our medical school. The anonymous questionnaire followed a multiple choice format divided into two sections: section 1 included student demographics and a self-assessment of knowledge of radiology compared with other medical subjects, as well as previous exposure to instruction and lectures/teaching in radiology. The second section (section 2) assessed awareness and general knowledge of radiation exposures associated with diagnostic imaging studies [14, 15]. Correct answers were awarded one mark, whereas an incorrect answer or omission received a mark of 0 . Results were stratified according to gender difference, previous exposure to instruction or lectures/teaching in diagnostic radiology, teaching in radiation protection and perceived knowledge of radiology.
Statistical analyses were performed using SPSS version 15.0 (2006, Chicago, IL). Histograms and the Shapiro-Wilk tests of normality were used to assess normal distribution of means. Chi-square tests of independence were used to analyse individual questions. Data were initially analysed using Student's t-tests, followed by Mann-Whitney U tests and Kruskall-Wallis tests. Statistical significance was defined at a p-value of less than 0.05 .

\section{Results}

The target population (Years 1-5 April 2008 and Year 0 September 2008) included 744 medical students. Of this group, 670 medical students completed the questionnaire (Appendix), giving a response rate of $90 \%$. Four hundred and two students were female and 268 were male, resulting in a $60 \%: 40 \%$ female:male ratio. Eighty-two percent of the study population compared with $4.1 \%$ of controls had been exposed to lectures or teaching in diagnostic radiology prior to completing the questionnaire. Despite this, $87 \%$ of the study population and $89 \%$ of controls considered that they had never been exposed to lectures or teaching focussed on radiation protection.

The study population performed much better than the control group with mean scores of $60 \%$ and $38 \%$, respectively $(p<0.001)$. Mean scores improved with the number of years completed successfully in medical school with each successive class outperforming the year below (e.g. Year 4 did better than Year 3, $p<0.001$ ) (Table 1). The greatest difference in mean scores was between 4 th and 5 th years $(65 \%$ versus $81 \%$ respectively, $\mathrm{p}<0.001)$. In addition, it was observed that students who received teaching in diagnostic radiology and radiation protection performed better than their counterparts who did not $(\mathrm{p}<0.001)$ (Table 2$)$. Graduate entry medical students tended to be more knowledgeable than their undergraduate colleagues (Tables 1 and 3).

Almost $50 \%$ of the control group thought the radiation dose of a chest radiograph was greater than 1 week of natural background radiation and $40 \%$ chose didn't know as the answer. Of the study population, $57 \%$ overestimated the amount of radiation in a chest radiograph, with $17.5 \%$ choosing the option that the radiation exposure would be equivalent to a dose greater than 1 year of background radiation. Thirty-seven percent of students in the study population correctly answered that an abdominal radiograph involved higher radiation exposure than a chest radiograph compared with only $16 \%$ of controls $(p<0.001)$.

Students in the study group had a moderate understanding of issues surrounding radiation dose associated with CT. Sixty-seven percent of the study population and only $39 \%$ of controls knew that CT involved the use of x-rays (Table 3). Almost $60 \%$ of 5 th year students knew that an 
Table 1 Mean scores for each year (from a total of 19 questions). Mean scores increased when each year was compared with the one below. Fifth year students achieved a mean score of $81 \%$. Students who entered medical school as postgraduates had higher scores compared with undergraduate students.

\begin{tabular}{lllll}
\hline Year & $\mathrm{n}$ & Mean score & Mean scores in \% & Standard deviation \\
\hline 0 & 111 & 6.89 & 36.2 & 3.415 \\
0 (Postgraduates) & 36 & 8.25 & 43.4 & 3.492 \\
1 & 112 & 8.36 & 44 & 3.310 \\
2 & 88 & 9.76 & 51.4 & 3.775 \\
3 & 113 & 11.03 & 58.1 & 3.804 \\
4 & 110 & 12.34 & 64.95 & 3.048 \\
5 & 100 & 15.36 & 80.84 & 2.038 \\
Total & 670 & & & \\
\hline
\end{tabular}

abdominal CT was equivalent to 300-1,000 chest radiographs, substantially higher than the average of $20 \%$ among the study group as a whole. Students who received teaching in radiology chose the correct option significantly more often than those who had not $(26.5 \%$ compared with $6.6 \%$, respectively, $\mathrm{p}<0.001)$. Almost one-third of the study population answered correctly that CT accounted for the majority of the medical radiation exposure received by the population compared with only $8 \%$ of the control group $(\mathrm{p}<0.001)$.

The assessment of students' knowledge of principles of MRI and ultrasound yielded disappointing results with $3.6 \%$ of the study population and $8.2 \%$ of the controls answering that ultrasonography exposed patients to ionising radiation (Table 3). Similarly, 14\% of the study population and $30 \%$ of the control group answered that MRI used $\mathrm{x}-$ rays (Table 3 ). It was somewhat re-assuring that only $2 \%$ of the most experienced students (year 5) answered that MRI involved the use of x-rays; however, $27 \%$ of 4 th years (penultimate year in medical school) thought MRI resulted in the highest radiation exposure when asked to choose between plain film radiography of the abdomen, ultrasound, conventional radiography and MRI. Consequently, students who did not recognise that a plain radiograph of the abdomen was associated with the greatest radiation dose among the four options were significantly less likely to know that MRI is not currently believed to be associated with increased cancer risk $(\mathrm{p}<0.001)$ (Fig. 1). There was a difference between female and male medical students regarding perception of MRI and ionising radiation with significantly more females than males thinking that MRI used $\mathrm{x}$-rays (20\% compared with $11 \%, \mathrm{p}=0.009$ ).

Regarding cancer risks, $70 \%$ of the study population were aware that $\mathrm{CT}$ potentially increased the lifetime cancer risk compared with only $34 \%$ of controls $(\mathrm{p}<0.001)$ (Fig. 1 and Table 4). Just over $80 \%$ of the study group, compared with $61 \%$ of controls, correctly answered that children were more sensitive to the effects of ionising radiation than adolescents, adults or the elderly. Ninety-six percent of 5 th years responded correctly. Fifty-one percent of the study group and $42 \%$ of controls answered correctly that the kidney was less sensitive to radiation than the thyroid, breast or gonads $(p=0.057)$. The relatively high radiosensitivity of breast tissue was not appreciated by $26 \%$ and $29 \%$ of students in years 4 and 5 , respectively.

In general, students did not appear to understand the mechanism of image generation in MRI, with $23 \%$ of the study population thinking that MRI increased lifetime risk of cancer compared with $31.8 \%$ of controls $(p<0.001)$ (Fig. 1). Of the study population, 76\% correctly answered that there was no increased risk of cancer associated with ultrasound, but only $52 \%$ of the control group thought so $(\mathrm{p}<0.001)$. No students in year 5 thought ultrasound increased the risk of cancer. Students in both groups who had received teaching/instruction in diagnostic radiology were less likely to associate ultrasound and MRI with an increased cancer risk compared with those who had not received teaching $(\mathrm{p}<0.001)$ (Table 5).
Table 2 Factors influencing students' overall scores.

\begin{tabular}{|c|c|c|c|}
\hline \multicolumn{2}{|l|}{ Factors affecting mean scores of students } & \multirow{2}{*}{$\begin{array}{l}\text { Mean scores in \% } \\
61.3 \\
42.8\end{array}$} & \multirow{2}{*}{$\frac{\text { P-value }}{<0.001}$} \\
\hline Teaching in diagnostic radiology & $\begin{array}{l}\text { Yes } \\
\text { No }\end{array}$ & & \\
\hline Education in radiation protection & $\begin{array}{l}\text { Yes } \\
\text { No }\end{array}$ & $\begin{array}{l}66 \\
53.3\end{array}$ & $<0.001$ \\
\hline Self-rated knowledge of radiology & $\begin{array}{l}\text { Excellent/good } \\
\text { Average/poor/none }\end{array}$ & $\begin{array}{l}76 \\
52.4\end{array}$ & $<0.001$ \\
\hline Self-rated knowledge of medical physics & $\begin{array}{l}\text { Excellent/good } \\
\text { Average/poor/none }\end{array}$ & $\begin{array}{l}63.2 \\
54.2\end{array}$ & 0.004 \\
\hline Gender & $\begin{array}{l}\text { Male } \\
\text { Female }\end{array}$ & $\begin{array}{l}56.4 \\
54.2\end{array}$ & $0.218^{*}$ \\
\hline
\end{tabular}


Table 3 Percentage of students in each year associating ionising radiation with various modalities. The figures in parentheses represent the student numbers. Students were most knowledgeable about plain film radiography. Imaging studies encountered less frequently by students such as mammography and barium studies were less well understood.

\begin{tabular}{|c|c|c|c|c|c|c|c|}
\hline Imaging study & Year 0 & Year 0 (postgrad) & Year 1 & Year 2 & Year 3 & Year 4 & Year 5 \\
\hline Chest radiograph & $91(101)$ & $100(100)$ & $91.1(111)$ & $98.9(86)$ & $99.1(112)$ & $99.1(109)$ & 99 (99) \\
\hline $\mathrm{CT}$ & $41.4(46)$ & $33.3(12)$ & $50(56)$ & $56.3(49)$ & $62.8(71)$ & $82.7(91)$ & $87(87)$ \\
\hline MRI & $29.7(33)$ & $11.1(4)$ & $24.1(27)$ & $16.1(14)$ & $10.6(12)$ & $16.4(18)$ & $2(2)$ \\
\hline Ultrasound & $9(10)$ & $5.6(2)$ & $4.5(5)$ & $3.4(3)$ & $5.3(6)$ & $3.6(4)$ & $1(1)$ \\
\hline Mammography & $36.9(41)$ & $55.6(20)$ & $44.6(50)$ & $54(48)$ & $77(87)$ & $74.5(82)$ & $82(82)$ \\
\hline Barium Studies & $36(40)$ & $38.9(14)$ & $46.4(52)$ & $71.3(63)$ & $71.7(81)$ & $82.7(91)$ & $88(88)$ \\
\hline Angiography & $25.2(28)$ & $22.2(8)$ & $44.6(50)$ & $62.1(54)$ & $69(78)$ & $80.9(89)$ & $81(81)$ \\
\hline
\end{tabular}

\section{Discussion}

The European Council issued the Eurotom directive issued in 1997 in an effort to address radiation protection awareness [6]. This directive states that radiation exposure for medical purposes should yield a net benefit to the patient and society. The use of alternative imaging techniques using less radiation or avoiding radiation altogether should be considered when clinical decisions are being made. Medical students represent future medical practitioners, and according to the directive unless they are taught which imaging methods use radiation and the approximate quantity of radiation involved they will be unable to make appropriate informed clinical decisions. This study demonstrates many important shortcomings in medical students' knowledge regarding important aspects of radiation protection that should be considered when developing the undergraduate medical curriculum to meet challenges of the future.
Student's on entry to medical school (year 0) were used as controls as they had yet to receive any teaching in radiology or radiation protection in a post-graduate setting. Graduate entry medical students (year 0) tended to be more knowledgeable than their undergraduate colleagues, probably due to their previous undergraduate studies, age and life experience, but this difference was not statistically significant. Although all students (years 1-5) were exposed to a 5-year undergraduate integrated curriculum that included instruction in clinical radiology, their understanding and knowledge of the basic concepts central to radiation protection left much room for improvement. This may be partially explained by the lack of a dedicated radiation protection module in the 5-year CICR syllabus.

In spite of the absence of a formal radiation protection module, it was reassuring to note that students' knowledge of radiation protection issues improved year on year, with those in the study population (years 1-5) performing significantly better than students in the control group (year 0). In addition, students who received radiology teaching $(87 \%)$ performed
Fig. 1 Student knowledge of the association between certain imaging modalities and an increased cancer risk. As observed previously, the more senior the student, the more likely he/she was to be correct. For example, 5 th years were more likely to know that ultrasound and MRI were not associated with an increase in cancer risk compared with 4 th years. A greater number of 5 th year students also knew that CT was associated with an increased risk of cancer compared with 4 th years.

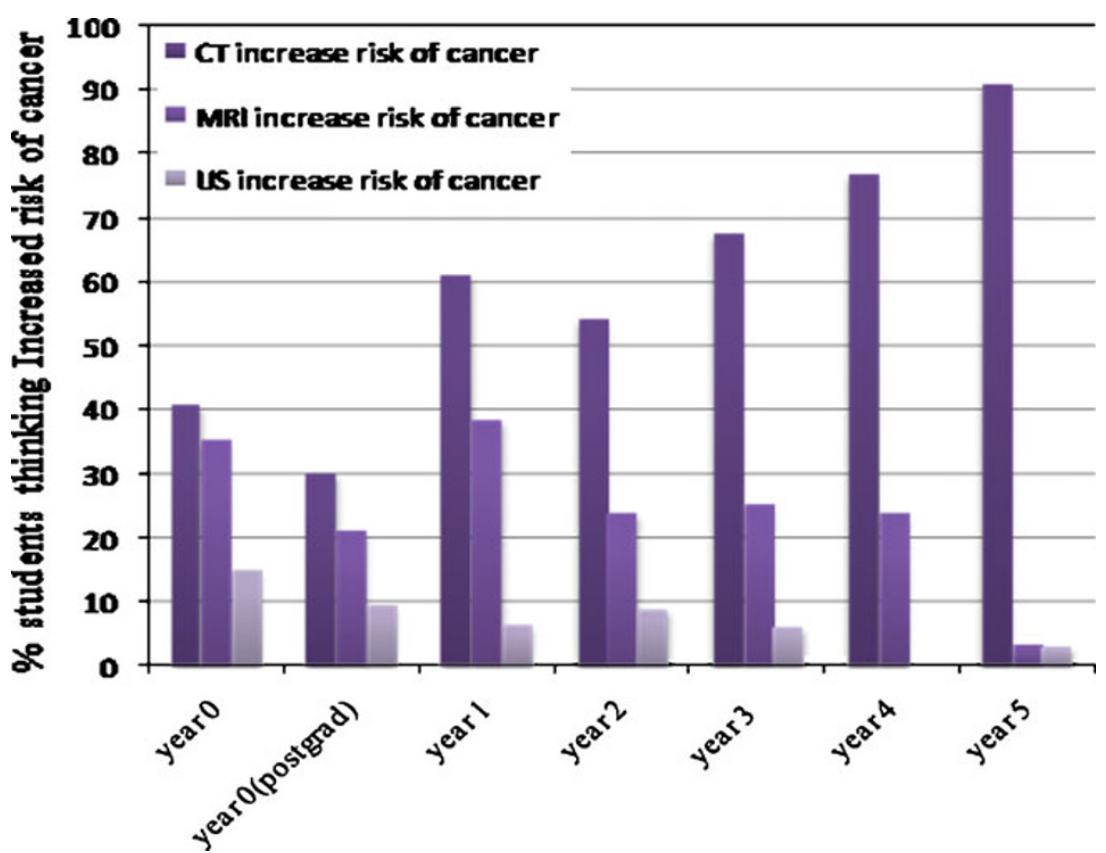


Table 4 Factors that influenced students' knowledge of the cancer risks associated with $\mathrm{CT}$. Teaching in radiology, radiation protection and knowledge were all associated with improved levels of knowledge.

\begin{tabular}{llll}
\hline Factors affecting perception & $\begin{array}{l}\% \text { associating CT } \\
\text { with cancer risk }\end{array}$ & P-value \\
\hline Teaching in radiology & Yes & 73.4 & $<0.001$ \\
& No & 44.5 & \\
Education in radiation & Yes & 74 & 0.031 \\
protection & No & 62 & \\
Knowledge of radiology & Excellent & 86 & $<0.001$ \\
& None & 27.2 & 0.007 \\
Knowledge of medical & Excellent & 95 & \\
physics & None & 51 & 0.266 \\
Gender & Male & 66.2 & \\
& Female & 56.4 & \\
\hline
\end{tabular}

significantly better those who had not. Undoubtedly, this was due to the many references to radiation protection issues in the CICR and "learning by osmosis" effect. The most significant improvement in performance was apparent between years 4 and 5. The superior performance of 5th year students undoubtedly reflected the contribution of the intensive clinical radiology teaching delivered to final-year medical students by clinical radiologists in preparation for their final exams. This teaching involved small group tutorials, case review sessions and formal lectures. Each student at our university is examined in interpretation of diagnostic imaging studies as part of the clinical component of the final medical examination. This likely positively impacts students' attendance for radiology teaching and the amount of time dedicated to radiology prior to final medical examination.

The fact that only two-thirds of the study population knew that CT used ionising radiation reflects an important gap in students' knowledge. The number of students that correctly

Table 5 Factors affecting students' understanding of MRI and its safety. Teaching in radiology, radiation protection and knowledge of radiology and physics were all associated with better levels of knowledge.

\begin{tabular}{llll}
\hline Factors affecting perception & & $\begin{array}{l}\text { \% associating } \\
\text { MRI with } \\
\text { cancer risk }\end{array}$ & P-value \\
\hline Teaching in radiology & Yes & 22.7 & $<0.001$ \\
& No & 26.5 & \\
Education in radiation & Yes & 16.6 & 0.031 \\
protection & No & 26.3 & \\
Knowledge of radiology & Excellent & 7.5 & $<0.001$ \\
& None & 31.2 & \\
Knowledge of medical & Excellent & 19.6 & 0.007 \\
physics & None & 25.1 & $0.277^{*}$ \\
Gender & Male & 21.8 & \\
& Female & 29 & \\
\hline
\end{tabular}

*The difference was significant in years 0,1 and 3 quantified the approximate radiation dose of a CT was disappointing, with only $60 \%$ of 5 th years knowing the correct answer. In designing the questionnaire, we chose not to interrogate students regarding the dose of diagnostic imaging studies in millisieverts. Instead, we focussed on radiation exposures relative to that of a postero-anterior chest radiograph, i.e. the dose of an abdominal CT was expressed in terms of an equivalent number of chest radiographs. In our opinion, quantifying $\mathrm{CT}$ doses in terms of an equivalent number of chest radiographs is a tangible method of quantifying the high radiation doses involved in CT imaging, and knowing relative values should be a prerequisite for medical students and referring physicians alike. The most poorly answered question concerned the radiation dose imparted by a chest radiograph. Less than $10 \%$ of students in both groups chose the correct answer (less than 1 week). It is hoped that greater awareness of these relative doses by the introduction of dedicated instruction in radiation protection may help minimise in the increasing number of unwarranted CT referrals in favour of lower dose modalities or imaging modalities such as ultrasound and MRI.

Much of the evidence regarding $\mathrm{CT}$ and cancer risk has been extrapolated from the nuclear fallout following the bombing of Hiroshima and Nagaski during World War II, and from longterm low-dose exposure to ionising radiation among nuclear radiation workers $[2,16]$. A lifetime cancer risk of 1 in 2,000 following one abdominal CT has generally been accepted for an adult [17]. This figure rises to 1 in 550 for children, due to their younger age and greater increased time for neoplasia to manifest itself [3]. It is reassuring that $70 \%$ of medical students in the study population were aware of the potential association between $\mathrm{CT}$ and an increased lifetime risk of cancer. In contrast, a recent study showed that $9 \%$ of emergency doctors and $47 \%$ of radiologists surveyed believed CT increased cancer risk [18]. Similar findings in a study published from Northern Ireland revealed that only $19 \%$ of doctors were aware of the association between medical radiation and increased cancer risk [19].

A disproportionate number of students in the study population incorrectly believed that MRI used ionising radiation. Furthermore, a quarter of students in the study population associated MRI with an increased risk of cancer. For the two questions above regarding MRI, females performed worse than males. The discrepancy in performance between males and females in correctly answering the questions regarding MRI was most notable among the control group. Interestingly, a recent study that investigated physicians' knowledge found similar differences between males and females with $39 \%$ of female and $19 \%$ of male doctors incorrectly responding that MRI used ionising radiation [20]. This is contrary to what one would expect given that MRI and ultrasound are preferentially performed during pregnancy in order to limit radiation exposure to the foetus [21].

This study demonstrated an incremental increase in students' knowledge of radiation protection with each year 
of medical teaching. Knowledge levels were highest among 5th year students, just prior to graduation and taking up positions as hospital interns. An important component of interns daily workload would be the ordering and organisation of diagnostic imaging studies under the supervision of senior attending physicians [22]. This highlights the importance of radiation protection instruction to medical undergraduates. While the majority of students were exposed to instruction in clinical radiology, over $80 \%$ of medical students considered that they had no teaching in radiation protection, and this deficiency in the curriculum was associated with poorer performance in the questionnaire. Exposure to formal teaching in clinical radiology, however, was associated with better performance in the questionnaire. Although only one university medical school was examined, the results of this study are still instructive to radiology educators. This study helps demonstrate that students acquire knowledge about radiation protection through teaching of clinical radiology. However, we now believe that some dedicated instruction in radiation protection, in the form of a focussed module, is required to ensure that the objectives of the Eurotom 97 directive are achieved and the clinicians of tomorrow are informed to the best of their ability, appropriate to their level of responsibility.

The average student in this study scored reasonably well, which contrasts with previous studies, which have found many deficiencies in practicing physicians' knowledge [6-8, 19]. This was probably because different standards of knowledge were required of doctors, and the questions asked of them were presented differently. The current study was designed with undergraduate students in mind, using closed ended questions. Previous studies have examined medical practitioners using open-ended questions requiring quantification of cancer risks and exact radiation doses of multiple imaging studies. Although it would be unfair to expect medical students to quantify exact doses, it is not unreasonable to expect them to know the forms of imaging that use radiation and the hierarchy of radiation exposure. Overall, the disappointing findings regarding student knowledge of many of the basic principles of radiation protection were interpreted as being due to a lack of formal focussed teaching/instruction in radiation protection.

In conclusion, this study demonstrates improved performance at multiple choice questionnaire designed to assess students' awareness of radiation exposures associated with diagnostic imaging investigations with increasing years spent in medical school and/or increasing exposure to instruction in clinical radiology. The findings likely support the Eurotom 97 directive stating that medical schools should implement radiation protection instruction as part of the undergraduate medical curriculum.

\section{Appendix}

Multiple choice questionnaire completed by each student. Students were asked about their background and education in radiology and radiation protection. Students' radiation knowledge was subsequently assessed.

\section{Multiple Choice Questionnaire}

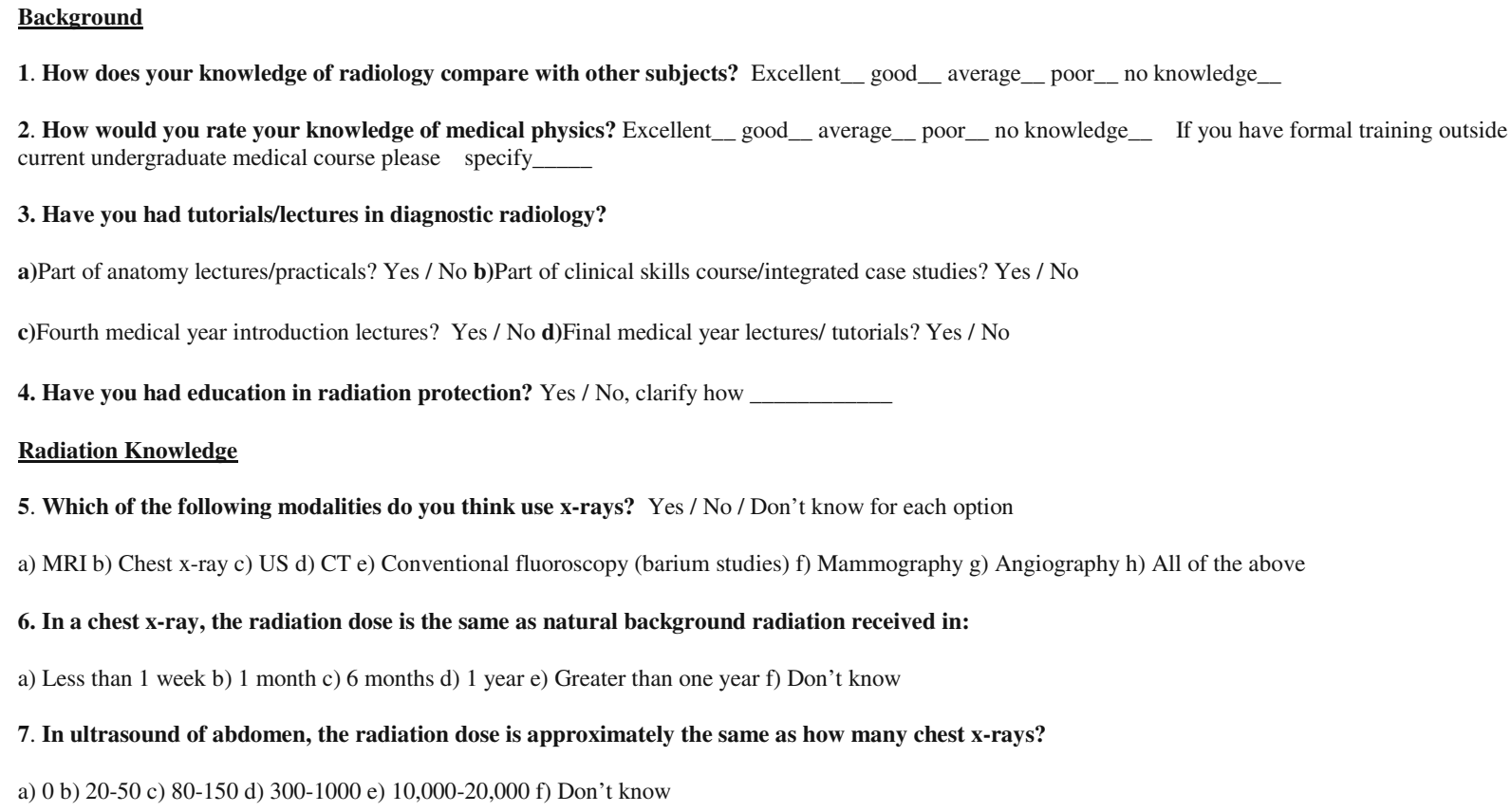


8. In CT of abdomen, the radiation dose is approximately the same as how many chest x-rays?

a) 0 b) $20-50$ c) $80-150$ d) 300-1000 e) 10,000-20,000 f) Don't know

9. In MRI of abdomen, the radiation dose is approximately the same as how many chest x-rays?

a) 0 b) $20-50$ c) $80-150$ d) 300-1000 e) 10,000-20,000 f) Don't know

10. Which of the following involves the highest radiation exposure for the patient?

a) Ultrasound of abdomen b) Plain film of abdomen c) MRI of spine d) Chest X-ray e) Don't know

11. Please circle which one of the following is most sensitive to radiation:

a) Children b) Adolescents c) Adults d) Elderly e) Don't know

12. Please circle which organ is least sensitive to radiation:

a) Thyroid b) Breast tissue c) Gonads d) Kidney e) Don't know

13. Medical imaging accounts for approximately $15 \%$ of the radiation dose received by the population. Which of the following modalities is responsible for most of this radiation dose?

a) Ultrasound b) Chest x-ray c) CT d) MRI e) Lumbar spine x-ray f) Don't know

14. Do you think any of the following increase the lifetime risk of developing cancer?

(Score each option using the following: 1=definitely yes, 2=probably yes, 3= probably no, 4=definitely no, 5=don't know) a) MRI b) Chest X-ray c) Ultrasound d) CT

\section{References}

1. Desmond A, O' Regan K, Curran C, McWiliams S, Fitzgerald T, Maher MM, Shanahan F (2008) Crohn's disease: factors associated with exposure to high levels of diagnostic radiation. Gut 57 (11):1524-1529

2. Cardis E, Vrijheid M, Blattner M, Gilbert E, Hakama M, Hill C et al (2007) The 15-country collaborative study of cancer risk among radiation workers in the nuclear industry: estimates of radiationrelated cancer risks. Radiat Res 167:396-416

3. Hart D, Wall BF (2004) UK population dose from medical X-ray examinations. Eur Radiol 50:285-291

4. Brenner D, Elliston C, Hall E, Berdon W (2001) Estimated risks of radiation-induced fatal cancer from pediatric CT. Am J Rad 176:289-296

5. Brenner D, Hall E, Phil D (2007) Computed tomography-an increasing source of radiation exposure. NEJM 357:322

6. Council Directive 97/43/Euratom of 30 June 1997 on health protection of individuals against the dangers of ionizing radiation in relation to medical exposure and repealing Directive 84/466/ Euratom. Official Journal of the European Communities09/07/ 1997; L180: 0022-0027

7. Sidhu MK, Goske MJ, Coley BJ, Connolly B, Racadio J, Yoshizumi TT, Utley T, Strauss KJ (2009) Image gently, step lightly: increasing radiation dose awareness in pediatric interventions through an international social marketing campaign. J Vasc Interv Radiol 20(9):1115-1159

8. Goske MJ, Applegate K, Boylan J et al (2008) The Image Gently campaign: working together to change practice. Am J Rad 190:273-274

9. Shiralkar S, Rennie A, Snow M, Galland RB, Lewis MH, GowerThomas K (2003) Doctors' knowledge of radiation exposure: questionnaire study. BMJ 327:371-372

10. Quinn AD, Taylor CG, Sabharwal T, Sikdar T (1997) Radiation protection awareness in non-radiologists. Br J Radiol 70:102-106
11. Jacob K, Vivian G, Steel JR (2004) X-ray dose training: are we exposed to enough? Clin Radiol 59:928-934

12. Tavakoli M, Seilanian Toosi F, Saadatjou SA (2003) Knowledge of medical students on hazards of ionizing radiation. J Med Educ 3(1):3-6

13. Mubeen MS, Abbas Q, Nisar N (2008) Knowledge about ionising and non-ionising radiation among medical students. J Ayub Med Coll Abbottabad 20(1):118-121

14. Health Physics Society. Available via http://www.hps.org/public information/asktheexperts.cfm. Accessed 12 Jun 2009

15. Notes for Guidance on the Clinical Administration of Radiopharmaceuticals and Use of Sealed Radioactive Sources, March 2006. The Administration of Radioactive Substances Advisory Committee. Available via http://www.arsac.org.uk/notes_for_ guidance/index.htm. Accessed Jun 122009

16. Berrington de González A, Darby S (2004) Risk of cancer from diagnostic X-rays: estimates for the UK and 14 other countries. Lancet 363:345-351

17. Wall BF, Hart D (1997) Revised radiation doses for typical X-ray examinations. Br J Radiol 70:437-439

18. Lee C, Haims AH, Monico E, Brink J, Forman H (2004) Diagnostic CT Scans: Assessment of Patient, Physician, and Radiologist Awareness of Radiation Dose and Possible Risks. Radiology 231:393-398

19. Soye JA, Paterson A (2008) A survey of awareness of radiation dose among health professionals in Northern Ireland. Br J Radiol 81(969):725-729

20. Arslanoğlu A, Bilgin S, Kubal Z, Ceyhan MN, Ilhan MN, Maral I (2007) Doctors' and intern doctors' knowledge about patients' ionizing radiation exposure doses during common radiological examinations. Diagn Interv Radiol 13(2):53-55

21. Kilpatrick CC, Orejuela FJ (2008) Management of the acute abdomen in pregnancy: a review. Curr Opin Obstet Gynecol 20 (6):534-539

22. Podnos YD, Williams RA, Jimenez JC, Stemmer EA, Gordon IL, Wilson SE (2003) Reducing the noneducational and nonclinical workload of the surgical resident: defining the role of the health technician. Curr Surg 60(5):529-532 